\title{
EL LOCUS STANDI DE TERCEROS ESTADOS PARA INTERPONER RECURSO DE ANULACIÓN CONTRA MEDIDAS RESTRICTIVAS DE LA UE: EL ASUNTO C-872/19 P, VENEZUELA/CONSEJO
}

\author{
Beatriz Vázquez Rodríguez ${ }^{1}$ \\ Universidad de Oviedo \\ vazquezbeatriz@uniovi.es
}

Cómo citar/Citation

Vázquez Rodríguez, B. (2021).

El locus standi de terceros Estados para interponer recurso de anulación contra medidas restrictivas de la UE: el asunto C-872/19 P, Venezuela/Consejo. Revista de Derecho Comunitario Europeo, 70, 1037-1060. doi: https://doi.org/10.18042/cepc/rdce.70.07

\section{Resumen}

La sentencia del Tribunal de Justicia de 22 de junio de 2021, Venezuela/Consejo, abre una nueva dimensión en relación con el alcance de los criterios de admisibilidad del art. 263 TFUE, párr. cuarto, puesto que el TJ considera por primera vez que Venezuela, un Estado tercero, debe considerarse "persona jurídica» y por tanto está legitimado — siempre que se cumplan los demás requisitos de admisibilidad — para plantear un recurso de anulación contra medidas restrictivas dirigidas contra él. Según el TJ, esta interpretación es acorde con el principio a la tutela judicial efectiva y

1 Profesora contratada doctora (acr.) de Derecho Internacional Público, Universidad de Oviedo. Miembro del Grupo de Investigación Consolidado de Derecho Europeo de la Universidad de Oviedo «EURODER-UNIOVI-IDI/2018/000187». El presente trabajo se adscribe al proyecto de I+D DER2017-86017-R, «Obstáculos a la movilidad de personas en los nuevos escenarios de la UE». 
el respeto del Estado de Derecho. Además, el interés de este asunto también radica en las argumentaciones en torno al alcance de principios de derecho internacional público como el de reciprocidad y el de inmunidad soberana. Finalmente, el fallo del TJ también permite aventurar la posibilidad de un cambio de paradigma en la elección y adopción de medidas restrictivas por la UE.

\section{Palabras clave}

Artículo 263 TFUE, párrafo cuarto; concepto de «persona jurídica»; locus standi; medidas restrictivas; Estado tercero.

\section{THE LOCUS STANDI OF THIRD STATES TO FILE AN ANNULMENT APPEAL AGAINST RESTRICTIVE MEASURES OF THE EU: CASE C-872/19 P, VENEZUELA/COUNCIL}

\section{Abstract}

The judgment of the Court of Justice of June 22, Venezuela/Council, opens a new dimension in relation to the scope of the admissibility criteria of art. 263 TFEU, fourth paragraph. For the first time, the Court has considered that Venezuela, a third State, should be considered a "legal person" and therefore is entitled — if the other admissibility requirements are met - to file an appeal for annulment against restrictive measures directed against it. According to the Court, this interpretation is in accordance with the principle of effective judicial protection and respect for the rule of law. In addition, the interest of this matter also lies in the arguments regarding the scope of principles of public international law such as reciprocity and sovereign immunity. Finally, the ruling of the Court of Justice also allows for the possibility of a paradigm shift in the choice and adoption of restrictive measures by the EU.

\section{Keywords}

Fourth paragraph of Article 263 TFEU; concept of a 'legal person'; locus standi; restrictive measures; action for annulment brought by a third State.

\section{LA QUALITÉ POUR AGIR DES ÉTATS TIERS POUR FORMER UN RECOURS EN ANNULATION CONTRE DES MESURES RESTRICTIVES DE L'UE: AFFAIRE C-872/19 P, VENEZUELA/CONSEIL}

\section{Résumé}

L'arrêt de la Cour de justice du 22 juin 2021, Venezuela/Conseil, ouvre une nouvelle dimension par rapport à la portée des critères de recevabilité de l'art. 263 
TFUE, quatrième alinéa puisque la $\mathrm{CJ}$ considère pour la première fois que le Venezuela, État tiers, doit être considéré comme une "personne morale» et est donc en droit - tant que les autres conditions de recevabilité sont remplies — d'introduire un recours en annulation contre les mesures restrictives à son encontre. Selon la Cour, cette interprétation est conforme au principe de protection juridictionnelle effective et au respect de l'État de droit. Par ailleurs, l'intérêt de cette affaire réside également dans les arguments relatifs à la portée de principes de droit international public tels que la réciprocité et l'immunité souveraine. Enfin, l'arrêt de la Cour de justice permet également d'oser la possibilité d'un changement de paradigme dans le choix et l'adoption de mesures restrictives par l'UE.

\section{Mots clés}

Article 263, quatrième alinéa; TFUE; notion de "personne morale»; qualité pour agir; mesures restrictives; recours en annulation introduit par un État tiers. 


\section{SUMARIO}

I. INTRODUCCIÓN. II. LOS CRITERIOS DE ADMISIBILIDAD DEL ARTÍCULO 263 TFUE, PÁRRAFO CUARTO: LO QUE EL TG NO QUISO VALORAR: 1. El concepto de «persona jurídica»: 1.1. Las alegaciones de las partes con respecto del concepto de «persona jurídica» en el recurso de casación. 1.2. La apreciación del TJ. 2. El interés en ejercitar la acción. 3. El margen de apreciación y la consideración de acto reglamentario que no incluye medidas de ejecución. III. LA PRESENCIA DEL DERECHO INTERNACIONAL EN LAS ARGUMENTACIONES: LO QUE EL TJ NO ENTRÓ A CONSIDERAR. IV. LA DIVERGENTE ARGUMENTACIÓN POR EL TG Y EL TJ DEL REQUISITO DE LA AFECTACIÓN DIRECTA CON RESPECTO A VENEZUELA. IV. LAS POSIBLES CONSECUENCIAS DE LA SENTENCIA. BIBLIOGRAFÍA.

\section{INTRODUCCIÓN}

El pasado mes de junio, el Tribunal de Justicia —a través de un recurso de casación y en contra de la posición previa adoptada por el Tribunal General en el asunto T-65/18 de 20 de septiembre de 2019² - decidió que la República Bolivariana de Venezuela estaba legitimada para impugnar un reglamento de la UE que establece medidas restrictivas contra ese Estado. La relevancia de la sentencia de 22 de junio de 2021 (Gran Sala) dictada en el asunto C-872/19 $P^{3}$ obedece a dos aspectos fundamentales. De un lado, como puso de relieve el abogado general en sus conclusiones, el recurso de casación «ofrece al Tribunal de Justicia una oportunidad excepcional para pronunciarse sobre la aplicación de los criterios de admisibilidad establecidos en el artículo 263 TFUE, párrafo cuarto" ${ }^{4}$ y el Tribunal la ha aprovechado, avanzando así en la construcción jurisprudencial de esta disposición para seguir perfilando su alcance, tarea en la que de nuevo el TJ muestra una cierta sensibilidad al adoptar una decisión acorde

2 Sentencia del Tribunal General de 20 de septiembre de 2019, Venezuela/Consejo, T-65/18, EU: T:2019:649.

3 Sentencia del Tribunal de Justicia de 22 de junio de 2021, Venezuela/Consejo, C-872/19 P, EU:C:2021:507.

4 Conclusiones del abogado general Hogan, Venezuela/Consejo, C-872/19 P, EU:C:2021:37, punto 2. 
al principio de tutela judicial efectiva en el marco de un recurso sumamente limitado a los demandantes particulares. De otro, al haber reconocido por primera vez el locus standi de terceros Estados para plantear recurso de anulación, no puede obviarse que esta situación puede suponer un efecto llamada para que otros Estados no miembros afectados por medidas restrictivas de la UE planteen recursos en el mismo sentido, lo que, como alegó el Consejo de la UE —atendiendo a principios de derecho internacional público- podría colocar a la Unión Europea en situación de desventaja dada la inexistencia de un derecho equivalente de revisión de posibles decisiones adoptadas por algunos Estados terceros contra esta organización supranacional.

Siendo así, el objeto de este comentario se centra en el análisis de la primera de las cuestiones, esto es, la valoración de los criterios de admisibilidad establecidos en el art. 263, párr. 4 TFUE con respecto al caso concreto y en particular, al alcance del concepto de "persona jurídica" de la citada disposición, dado que supone la cuestión más novedosa del presente asunto. Sin embargo, a pesar del carácter hipotético de la segunda cuestión planteada, en la conclusión final se tratará de poner de relieve si efectivamente este nuevo enfoque del TJ puede entrañar algún peligro para la UE o, por el contrario, obedece una garantía más con respecto de la tutela judicial efectiva que debe ser observada por una organización que consagra el Estado de Derecho como uno de sus valores fundamentales sobre los que se sustenta.

\section{LOS CRITERIOS DE ADMISIBILIDAD DEL ARTÍCULO 263 TFUE, PÁRRAFO CUARTO: LO QUE EL TG NO QUISO VALORAR}

Como es sabido, ante la situación de inestabilidad política y económica y el creciente deterioro de la democracia y el Estado de Derecho en Venezuela, el 13 de noviembre de 2017 el Consejo de la UE adoptó la Decisión 2017/20174 relativa a medidas restrictivas habida cuenta de la situación en Venezuela ${ }^{5}$ y el

5 Decisión (PESC) 2017/20174 del Consejo de 13 de noviembre de 2017, relativa a medidas restrictivas habida cuenta de la situación en Venezuela, (DO L 295, de 14 de noviembre de 2017, p. 60). Esta decisión contenía la prohibición de exportar a Venezuela armas, equipos militares o cualquier otro equipo que pudiese ser utilizado para la represión interna; la prohibición de prestar a ese Estado servicios financieros, técnicos o de otro tipo relacionados con esos equipos y tecnologías; y la inmovilización de fondos y recursos económicos de personas, entidades y organismos. Además, según su art. 13 esta decisión se aplicaría hasta el 14 de noviembre de 2018, por lo que el 6 de noviembre de 2018 el Consejo adoptó la Decisión (PESC) 2018/1656, por la que se modifica la Decisión 2017/2074 (DO L 276, de 7 de noviembre de 2018, 
Reglamento 2017/2063 . En particular, los arts. 2 y 3 del citado reglamento consagraron la prohibición de vender o suministrar, a cualquier persona física o jurídica, entidad u organismo en Venezuela, equipos militares y las tecnologías relacionadas con ellos que pudiesen utilizarse para la represión interna. Así mismo, se estableció la prohibición de prestar a esas mismas personas físicas o jurídicas, entidades u organismos en Venezuela determinados servicios técnicos, de intermediación o financieros relacionados con el suministro de dichos equipos. Finalmente, en los arts. 6 y 7 del citado reglamento se contempla la prohibición de venta, suministro o exportación de equipos o tecnología — procedentes o no de la UE — a cualquier persona, entidad u organismo sitos en Venezuela.

Como consecuencia de lo anterior, el 6 de febrero de 2018 la República Bolivariana de Venezuela interpuso un recurso de anulación contra el Reglamento 2017/2063. Posteriormente, mediante escrito separado presentado el 17 de enero de 2019, y en base al art. 86 del Reglamento de Procedimiento, la demandante amplió el recurso al Reglamento de ejecución (UE) 2018/1653 y a la Decisión (PESC) 2018/1656 en la medida en que consideró que sus disposiciones también le afectaban. Sin embargo, al declarar la inadmisibilidad del recurso con respecto de las disposiciones del reglamento por considerar que no afectaban directamente a la situación jurídica de Venezuela, el TG declaró también la inadmisibilidad del recurso con respecto de aquellos actos. El Consejo de la UE invocó tres motivos de inadmisibilidad ante el TG. En primer lugar, que Venezuela no tenía interés en ejercitar la acción; en segundo lugar, que las disposiciones impugnadas no afectaban directamente a ese Estado y, en tercer lugar, que Venezuela tampoco era una persona física o jurídica a efectos del art. 263 TFUE, párr. cuarto. No obstante, el TG consideró que debía pronunciarse únicamente sobre la admisibilidad del recurso examinando el segundo motivo, y en su fallo este tribunal estimó que las citadas disposiciones efectivamente no afectaban directamente a la demandante y declaró la inadmisibilidad del recurso en su totalidad. Siendo

p. 10), y prorrogó su validez hasta el 14 de noviembre de 2019 además de modificar el anexo I, que se refiere a una de las personas afectadas por la inmovilización de activos financieros.

6 Reglamento (UE) 2017/2063 del Consejo de 13 de noviembre de 2017, relativo a medidas restrictivas habida cuenta de la situación de Venezuela (DO L 295, de 14 de noviembre de 2017, p. 21), adoptado sobre la base del art. 215 TFUE. Posteriormente, al Consejo adoptó el Reglamento de ejecución (UE) 2018/1653 de 6 de noviembre de 2018 por el que se aplica el Reglamento (UE 2017/20163 (DO L 276, de 7 de noviembre de 2018, p. 1) que modificó IV del citado reglamento, que se refiere a una de las personas afectadas por la inmovilización de activos financieros. 
así, la República Bolivariana de Venezuela planteó un recurso de casación al TJ solicitando la anulación de la STG de 20 de septiembre de 2019.

Recordando que según reiterada jurisprudencia puede pronunciarse de oficio si es necesario sobre el motivo de orden público basado en el incumplimiento de los requisitos de admisibilidad establecidos en el art. 263 TFUE, el TJ decide abordar con carácter previo si Venezuela debe considerarse «persona jurídica» «dado que la respuesta a esta cuestión es necesaria para el examen del segundo motivo de inadmisibilidad invocado por el Consejo, que se discute en el marco del motivo único del recurso de casación y según el cual los artículos 2, 3, 6 y 7 del Reglamento 2017/2063 no afectan directamente a la República Bolivariana de Venezuela» ${ }^{7}$. Una vez aclarada esta cuestión, el TJ también examinará los demás requisitos de admisibilidad contemplados en el art. 263 TFUE, párr. cuarto. De todos ellos nos ocuparemos a continuación, centrándonos en la discusión en torno al concepto de persona jurídica, que como hemos apuntado más arriba es una de las cuestiones más relevantes del presente asunto.

\section{EL CONCEPTO DE «PERSONA JURÍDICA»}

A través de su decisión de 7 de julio de 2020, el TJ instó a las partes en el recurso de casación a pronunciarse sobre si un Estado tercero debe considerarse «persona jurídica» según el art. 263 TFUE, párr. cuarto y remitió una petición similar a la Comisión Europea y a los Estados miembros.

\subsection{Las posiciones con respecto del concepto de "persona jurídica» en el recurso de casación}

Venezuela sostuvo que del tenor del art. 263 TFUE, párr. cuarto, ni el objetivo o el contexto de la disposición permiten excluirla — ni siquiera indirectamente- del concepto de "persona jurídica». En ese sentido, se remitió al auto de 10 de septiembre de 2020, Camboya y CRF/Comisión, donde el TG había afirmado que «ni el artículo 263 TFUE, párrafo cuarto, ni ninguna otra disposición del Derecho primario excluyen a los Estados terceros del derecho a interponer un recurso de anulación", y concluyó que "como el texto de los Tratados no excluye a los Estados terceros, a estos, que gozan de personalidad jurídica tanto en el Derecho internacional como en el interno, no se les puede impedir impugnar un acto de la Unión ante el Tribunal si se cumplen

Sentencia del Tribunal de Justicia de 22 de junio de 2021, Venezuela/Consejo, C-872/19 P, EU:C:2021:507, apdo. 23. 
los requisitos exigidos por el artículo 263 TFUE, párrafo cuarto» ${ }^{8}$. Por todo ello este Estado interpretaba que el término "toda persona física o jurídica» de aquella disposición comprende a Estados no miembros y que excluirla de ese concepto supondría una interpretación contra legem que además también vulneraría la jurisprudencia cuyo punto de partida se encuentra en el asunto Plaumann/Comisión, según la cual «las disposiciones del Tratado relativas a la legitimación activa de los justiciables no pueden interpretarse de un modo restrictivo; y que, por tanto, ante el silencio del Tratado, no cabe deducir una limitación a este respecto»?. Finalmente, la recurrente añadió que la propia interpretación literal del art. 263 TFUE, párr. cuarto, se ve confirmada por el contexto normativo y la finalidad perseguida por la citada disposición puesto que el objetivo de aquella es garantizar una adecuada tutela judicial a todas las personas, físicas o jurídicas, afectadas directa e individualmente por los actos de las instituciones, un principio general del derecho de la UE que además está consagrado en el art. 47.1 de la Carta de los Derechos Fundamentales de la UE y en el art. 19.1 TUE ${ }^{10}$.

Por su parte, el Consejo de la UE mantuvo una posición totalmente opuesta y refutó la argumentación de Venezuela. Según esta institución, el no reconocer acceso a esta a los tribunales no es contrario al principio de tutela judicial efectiva contemplado en el art. 47 de la Carta, puesto que los Estados terceros no están sujetos al sistema del Derecho de la UE y, por ende, no son destinatarios de esta disposición. Para el Consejo, reconocer lo contrario implicaría extender las vías de recurso existentes en el actual sistema de recursos previsto por el propio Derecho de la UE, corriéndose el peligro de que los Estados terceros puedan utilizar «los órganos jurisdiccionales de la Unión como una puerta trasera para resolver conflictos internacionales entre sujetos de Derecho internacional público»" ${ }^{11}$.

En las posiciones de los Estados miembros ${ }^{12}$ también se pueden contemplar posiciones antagónicas sobre el concepto de "persona jurídica» a

8 Auto del Tribunal General de 10 de septiembre de 2020, Camboya y CRF/Comisión, T-246/19, EU: T:2020:415, apdos. 49 y 50.

9 Sentencia del Tribunal de Justicia de 15 de julio de 1963, Plaumann/Comisión, C-25/62, EU:C:1963:17.

10 Sentencia del Tribunal de Justicia de 22 de junio de 2021, Venezuela/Consejo, C-872/19 P, EU:C:2021:507, apdos. 25 a 29.

11 Sentencia del Tribunal de Justicia de 22 de junio de 2021, Venezuela/Consejo, C-872/19 P, apdo. 29.

12 Los Estados que presentaron observaciones sobre esta cuestión fueron: el Reino de Bélgica, la República de Bulgaria, la República Federal de Alemania, la República de Estonia, la República Helénica, la República de Lituania, el Reino de los Países Bajos, 
efectos del art. 263 TFUE, párr. cuarto. Mientras que algunos Estados (Grecia, Polonia, Eslovenia y Suecia) consideraron que un Estado no miembro no puede estar comprendido en ese concepto dado que el mismo se limita a las entidades dotadas de personalidad jurídica en virtud del derecho de un Estado miembro o de un Estado tercero, otros (como Bélgica, Bulgaria, Alemania, Estonia, Letonia, Lituania y Países Bajos) opinaron que un Estado tercero sí está comprendido en el concepto de "persona jurídica» ya que estos están dotados de personalidad jurídica según el derecho internacional público.

Finalmente, la Comisión Europea sostuvo que de una interpretación literal de aquella disposición no se podía extraer una conclusión definitiva. En ese sentido, entendió que los Estados terceros únicamente podrían considerarse como "persona jurídica» si tuviesen acceso al TJUE en virtud de un acuerdo internacional celebrado con la UE o, atendiendo a la doctrina de inmunidad de jurisdicción, cuando los actos que realizase ese Estado tercero pudieran catalogarse como iure gestionis y puesto que en el caso de Venezuela los actos realizados están vinculados al ejercicio del poder público, no podrían considerarse como tal. Según la Comisión, su postura es la más coherente con el principio de tutela judicial efectiva puesto que no niega el recurso a un Estado tercero, sino que queda garantizado en cada caso por la jurisdicción competente. De todas formas, la Comisión subraya que el tercer Estado debe cumplir los requisitos de afectación directa e individual establecidos en el derecho primario y en la jurisprudencia del TJ.

\subsection{La apreciación del TJ}

El TJ parte de la base de que para examinar si un Estado tercero puede considerarse "persona jurídica» a efectos del párr. cuarto del art. 263 TFUE, esta noción debe entenderse como un concepto autónomo del derecho de la UE para el cual no se hace remisión a los derechos nacionales puesto que, como ya había dicho el Tribunal, "the meaning of "legal person" in the second paragraph of Article 173 of the EEC Treaty is not necessarily the same as in the various legal systems of the Member States» ${ }^{13}$. Además, tal como dictamina su propia jurisprudencia, para la interpretación de este concepto de la citada disposición habrá que tener en cuenta no solamente el tenor literal de aquella disposición, sino también el contexto en el que se inscribe y los

la República de Polonia, la República de Eslovenia, la República Eslovaca y el Reino de Suecia.

13 Sentencia del Tribunal de Justicia de 28 de octubre de 1982, Groupement des Agences de voyages, Asbl/Comisión, 135/81, EU:C:1982:371, apdo. 10. 
objetivos perseguidos por la normativa de la que forma parte ${ }^{14}$. Pasando a analizar ambos aspectos, en lo que se refiere al tenor literal de la disposición el TJ entiende que ni el art. 263 TFUE, párr. cuarto, ni ninguna otra disposición de derecho primario contienen una definición de "persona jurídica», y puesto que no puede ser un concepto objeto de interpretación restrictiva, no puede negarse la posibilidad a que ciertas categorías de personas puedan invocar su legitimación activa ante los órganos jurisdiccionales de la UE. En lo que se refiere a la segunda cuestión, el TJ apunta a su jurisprudencia sobre el particular, la cual demuestra que la legitimación activa en el recurso de anulación con respecto de estas categorías se ha ido extendiendo a otras al compás del contexto normativo y la finalidad perseguida por el art. 263 TFUE, párr. cuarto. Esta ampliación ha supuesto que se incluyan en el concepto de "persona física o jurídica» a entidades de carácter privado ${ }^{15}$ pero también de carácter público ${ }^{16}$ como entidades subestatales: regiones, comunidades o provincias, entre otras ${ }^{17}$ (Martín Baumeister, 2004: 29-45; Condinanzi y Mastroianni, 2009: 109). Así, como apuntara Faramiñán Gilbert, si bien al hablar de personas jurídicas se está haciendo referencia a personas jurídicas de derecho privado y de derecho público, «habrá que tener en cuenta la posibilidad de considerar personas jurídicas también, en ciertas circunstancias, a terceros Estado u Organizaciones Internacionales» (2007: 363).

En este sentido y con el objetivo de aclarar por primera vez esta posibilidad de si un tercer Estado puede considerarse una "persona jurídica» a los

14 Sentencia del Tribunal de Justicia de 6 de octubre de 2020, Jobcenter Krefeld, C181/19, EU:C:2020:794, apdo. 61.

15 El TJ se remite al Auto del Tribunal de Justicia de 26 de noviembre de 2009, Região autónoma dos Açores/Consejo, C-444/08 P, no publicado, EU:C:2009:733, apdo. 31; al Auto del Tribunal de Justicia de 1 de octubre de 1997, Regione Toscana/Comisión, C-180/97, EU:C:1997:451, apdos. 10 a 12, y a la Sentencia del Tribunal de Justicia de 22 de noviembre de 2001, Nederlandse Antillen/Consejo, C-452/98, EU:C:2001:623, apdos. 51.

16 En este sentido se refiere a la Sentencia del Tribunal de Justicia de 1 de febrero de 2018, Deutsche Bahn y otros/Comisión, C-264/16 P, no publicada, EU:C:2018:60, apdo. 2 y a la Sentencia del Tribunal de Justicia de 4 de febrero de 2020, Uniwersytet Wrocławski y Polonia/REA, C-515/17 P y C-561/17 P, EU:C:2020:73, apdo. 69.

17 Con respecto a esta inclusión, el TJ en los apdo. 44 y 45 se refiere entre otras a: el Auto del Tribunal de Justicia de 26 de noviembre de 2009, Região autónoma dos Açores/Consejo, C-444/08 P, no publicado, EU:C:2009:733, apdo. 31; al Auto del Tribunal de Justicia de 1 de octubre de 1997, Regione Toscana/Comisión, C-180/97, EU:C:1997:451, apdos. 10 a 12; y a la Sentencia del Tribunal de Justicia de 22 de noviembre de 2001, Nederlandse Antillen/Consejo, C-452/98, EU:C:2001:623, apdos. 51. 
efectos del art. 263 TFUE, párr. cuarto, el AG apeló, a pesar de la novedad, a la existencia de una tendencia previa del TJUE en la que apoyarse para responder favorablemente a esa cuestión. Así, el AG se remitía - entre otros- al ya citado auto de 10 de septiembre de 2020, Camboya y CRF/Comisión, donde el TG había declarado que la expresión «toda persona física o jurídica» que figura en el art. 263 TFUE, párr. cuarto, debía entenderse en el sentido de que abarca también a los Estados no miembros de la Unión y al auto de 14 de julio de 2005, Suiza/Comisión, donde había llegado a la misma conclusión; no obstante, es importante poner de relieve que estas decisiones no se dictaron en un contexto de medidas restrictivas adoptadas por la UE, lo que sin embargo sí ocurría en el también citado asunto $P K K y K N K /$ Consejo. El TJ, apoyándose en las conclusiones del AG, considera fundamental traer a colación ese asunto puesto que el TJ había admitido que una organización sin personalidad jurídica como el Partido de los Trabajadores del Kurdistán (PKK) estaba legitimada para impugnar las medidas restrictivas que se le habían impuesto: si tenían entidad suficiente para ser destinatarios de las mismas, «la coherencia y la justicia imponen que se reconozca que dicha entidad también tiene existencia suficiente para impugnar dichas medidas ${ }^{18}$. De esta forma, este asunto no debía de haber sido ignorado por el TG, puesto que como apuntó el $A G$, "parece sugerir que no es necesario cumplir escrupulosamente el requisito de ser una "persona física o jurídica" para que una entidad pueda impugnar las medidas restrictivas con arreglo al artículo 263 TFUE, párrafo cuarto». Por ello, «cabe aducir que una entidad como la recurrente, siempre que pueda demostrar, en particular, que está directa e individualmente afectada por las medidas restrictivas, debe tener acceso a los tribunales de la Unión para proteger sus derechos, independientemente de la calificación jurídica que le confiera el Derecho nacional, el Derecho internacional o incluso el Derecho de la Unión» ${ }^{19}$.

En síntesis, esta cuestión parece estar en consonancia con la propia evolución jurisprudencial del TJ, tal como se ha apuntado arriba, ya que si se ha reconocido que organizaciones sin personalidad jurídica ${ }^{20}$ tienen la posibilidad de interponer recurso de anulación, no tendría justificación aparente

18 Sentencia del Tribunal de Justicia de 22 de junio de 2021, Venezuela/Consejo, C-872/19 P, apdo. 47. El TJ se refiere a la Sentencia del Tribunal de Justicia 18 de enero de 2007, PKK y KNK/Consejo, C-229/05 P, EU:C:2007:32, apdo. 112.

19 Conclusiones del abogado general Hogan, Venezuela/Consejo, C-872/19 P, punto 79.

20 Precisamente, con carácter reciente y citando expresamente la decisión del TJ objeto de este comentario, el TG ha reconocido que el Frente Popular para la Liberación de Saguía el Hamra y Río de Oro (Frente Polisario) es una persona jurídica en el sentido del art. 263 TFUE, párr. cuarto: Sentencia del Tribunal General de 29 de septiembre de 2021, Front Polisario/Consejo, T-279/19, EU: T:2021:639, apdos. 86 y 87. 
privar a un Estado tercero que también estuviese afectado por medidas restrictivas. Así, el concepto de persona jurídica no puede interpretarse de manera restrictiva. No obstante, ya se han apuntado algunos temores sobre si el reconocimiento de esta capacidad procesal de Estados terceros para acudir ante los órganos jurisdiccionales de la UE podría tener consecuencias negativas con respecto del estatuto del Estado en el derecho internacional, en el sentido de que este reconocimiento pudiera colocar a este tribunal en una posición incómoda para decidir sobre cuestiones de alto contenido político y muy vinculadas a la doctrina de reconocimiento de Estados en situaciones como las de Kosovo o Palestina (Kassoti, 2021) ${ }^{21}$.

Desde una interpretación contextual y teleológica del art. 263 TFUE, párr. cuarto, el TJ también aboga por reconocer a un tercer Estado como "persona jurídica» a los efectos del art. 263 TFUE, párr. cuarto, en contra de la posición del Consejo según la cual iría en contra del sistema de recursos previsto por el derecho de la UE. Para el TJ, el principio de tutela judicial efectiva y, por ende, la existencia de un control judicial efectivo para garantizar el cumplimiento de las disposiciones del derecho de la Unión, es inherente a la existencia del respeto al Estado de Derecho, un valor sobre el que se fundamenta la UE en virtud del art. 2 del TUE puesto que «dicha persona jurídica de Derecho internacional público, como cualquier otra persona o entidad, puede verse afectada negativamente en sus derechos o intereses por un acto de la Unión y, por lo tanto, debe poder solicitar, cumpliendo los referidos requisitos, la anulación de tal acto»»22.

En este sentido, el TJ —a diferencia de la posición del TG— ha preferido una interpretación más en consonancia con el principio a la tutela judicial efectiva puesto que si la adopción y aplicación de estas medidas «deben respetar los derechos humanos y las libertades fundamentales, en particular las debidas garantías procesales y el derecho a la tutela judicial efectiva» ${ }^{23}$, en un contencioso en el que cada vez existe una mayor litigiosidad hasta ahora limitada a los particulares ${ }^{24}$ este tipo de medidas deben estar en plena conformidad

21 Conviene advertir que la propia autora califica esos temores de exagerados.

22 Sentencia del Tribunal de Justicia de 22 de junio de 2021, Venezuela/Consejo, C-872/19 P, apdo. 50.

23 Orientaciones sobre la aplicación y evaluación de las medidas restrictivas (sanciones) en el marco de la Política Exterior y de Seguridad Común de la UE, Consejo de la UE, n. ${ }^{\circ}$ 5664/18, 4 de mayo de 2018, apdo. 9. Sobre esta cuestión véase Jiménez García (2017: 515).

24 Véanse por ejemplo: Sentencia del Tribunal de Justicia de 6 de octubre de 2020, Bank Refah Kargaran/ Consejo, C-134/19 P, EU:C:2020:793; Sentencia del Tribunal de Justicia de 6 de septiembre de 2018, Bank Mellat/Consejo, C-430/16 P, EU:C:2018:668; 
con la jurisprudencia de los tribunales de la UE. De esta forma, la jurisprudencia del TJUE no excluye la posibilidad de que se reconozca a una entidad capacidad procesal ante los órganos jurisdiccionales de la Unión con independencia de su personalidad jurídica de derecho interno, si los requisitos de tutela judicial efectiva así lo exigen. Así, puesto que el TJUE ha afirmado que "toda inscripción en una lista de personas o entidades contempladas por medidas restrictivas, ya esté basada en el artículo 215 TFUE o en el artículo 291 TFUE, apartado 2, permite a dicha persona o entidad, por cuanto le es equiparable a una decisión individual, acceder al juez de la Unión, conforme al artículo 263 TFUE, párrafo cuarto ${ }^{25}$, no debería impedirse a un Estado tercero contra el que se dirigen esas medidas una vía de recurso contra estas.

Como se ha puesto de relieve en relación con la limitada legitimación del particular en el recurso de anulación

Aunque puede ser cierto que el sistema comunitario languidece a veces para proteger eficazmente el de derecho a una tutela judicial efectiva, no puede negarse la evolución llevada a cabo. Sería en efecto, excesivo considerar que la apertura de las condiciones de admisibilidad del recurso de anulación del particular nunca formó parte de las preocupaciones del juez de la Unión. En realidad esa preocupación siempre existió, aunque fue manifestándose en la jurisprudencia de forma cuidadosamente circunscrita y progresiva. (Cortés Martín, 2014: 6)

Sentencia del Tribunal de Justicia de 28 de noviembre de 2013, Consejo/Manufacturing Support \& Procurement Kala Naft, C-348/12 P, EU:C:2013:776; Sentencia del Tribunal de Justicia de 28 de noviembre de 2013, Consejo/ Fulmen y Mahmoudian, C-280/12 P, EU:C:2013:775; Sentencia del Tribunal General de 12 de febrero de 2020, Mende Omalanga/Consejo, T-176/18, EU:T:2020:61; Sentencia del Tribunal General de 12 de febrero de 2020, Ruhorimbere/Consejo, T-175/18, EU:T:2020:62; Sentencia del Tribunal General de 12 de febrero de 2020, Ramazani Shadary/Consejo, T-173/18, EU:T:2020:48; Sentencia del Tribunal General de 31 de mayo de 2018, Kaddour/ Consejo, T-461/16, EU:T:2018:316; Sentencia del Tribunal General de 14 de marzo de 2018, Su Kim y otros/Consejo y Comisión, T-533/15, EU:T:2018:138; Sentencia del Tribunal General de 14 de marzo de 2017, Bank Tejarat/Consejo, T-346/15, EU:T:2017:164; Sentencia del Tribunal General de 13 de septiembre de 2018, VTB Bank/ Consejo, T-734/14, EU:T:2018:542, Sentencia del Tribunal General de 13 de septiembre de 2018, NK Rosneft OAO y otros/Consejo, T-715/14, EU:T:2018:544, Sentencia del Tribunal General de 15 de septiembre de 2016, Yanukovych/Consejo, T-348/14, EU:T:2016:508, Sentencia del Tribunal General de 14 de abril de 2016, Ben Ali/Consejo, T-200/14, EU:T:2016:216; Sentencia del Tribunal General de 3 de mayo de 2016, Post Bank Iran/Consejo, T-68/14, EU:T:2016:263.

25 Sentencia del Tribunal de Justicia de 1 de marzo de 2016, National Iranian Oil Company/Consejo, C-440/14 P, apdo. 44. 
En esta ocasión, al TJ le ha tocado pronunciarse sobre la situación de Estados terceros, y al hacerlo favorablemente su postura es acorde con la flexibilidad de las condiciones de admisibilidad que ha sido una constante en la jurisprudencia del TJ sobre el particular.

\section{EL INTERÉS EN EJERCITAR LA ACCIÓN}

Sobre el requisito del interés en ejercitar la acción, en el recurso de casación el Consejo alegó que según el art. 20 del reglamento el ámbito de aplicación de este se limitaba al territorio de los Estados miembros y a las personas sometidas a la jurisdicción de un Estado miembro. En este sentido, según la opinión del Consejo, había que aplicar por analogía el asunto Consejo/ Frente Polisario, y, en consecuencia, no podría considerarse que Venezuela estuviera legitimada para solicitar la anulación de la decisión impugnada. Según el TJ, recordando su jurisprudencia previa, «la existencia del interés en ejercitar la acción supone que la anulación de un acto impugnado pueda procurar, por sí misma, un beneficio a la persona física o jurídica que haya interpuesto el recurso ${ }^{26}$. Además, el TJ no comparte la aplicación del asunto en cuestión al caso concreto, puesto que en el caso Consejo/Frente Polisario si bien el Frente Polisario alegó que el Acuerdo en forma de Canje de Notas entre la Unión Europea y el Reino de Marruecos sobre medidas recíprocas de liberalización del comercio de productos agrícolas, productos agrícolas transformados, pescado y productos de la pesca, se aplicaba en el territorio del Sáhara Occidental, el TJ entendió lo contrario. En el caso presente, las disposiciones de este reglamento sí se adoptaron contra Venezuela, impidiendo a este tercer Estado llevar a cabo distintas actividades.

\section{EL MARGEN DE APRECIACIÓN Y LA CONSIDERACIÓN DE ACTO REGLAMENTARIO QUE NO INCLUYE MEDIDAS DE EJECUCIÓN}

En lo que se refiere a los últimos requisitos del test de admisibilidad del art. 263 TFUE, párr. cuarto, al considerar que las disposiciones impugnadas no afectaban a la situación jurídica de Venezuela el TG no entendió necesario valorar el segundo de los requisitos de afectación directa establecidos en la jurisprudencia del TJUE, esto es, que las disposiciones impugnadas no dejen ningún margen de apreciación a los destinatarios encargados de su aplicación,

26 Sobre este requisito, el TJ se refiere concretamente a la reciente Sentencia del Tribunal de Justicia de 21 de enero de 2021, Alemania/Esso Raffinage, C-471/18 P, EU:C:2021:48, apdo. 103. 
la cual debe tener carácter meramente automático y derivarse únicamente de la normativa de la Unión, sin aplicación de otras normas intermedias. Pues bien, para el TJ, de los arts. 2, 3, 6 y 7 del Reglamento 2017/2063 se desprende que las prohibiciones establecidas en estas disposiciones se aplican sin dejar margen alguno de apreciación a los destinatarios encargados de su aplicación y sin necesidad de adoptar medidas de ejecución, ni por la Unión ni por los Estados miembros.

Finalmente, en cuanto a la consideración de acto reglamentario, el TJ afirma que el reglamento en cuestión se adoptó sobre la base del art. 215 TFUE, y según la jurisprudencia del TJUE, «debe entenderse que el concepto de «acto reglamentario» en el sentido del artículo 263 TFUE, párrafo cuarto, incluye cualquier acto de carácter general a excepción de los actos legislativos ${ }^{27}$ y quedaba probado que, en este caso, la adopción del mismo emanaba de un acto legislativo. Por lo demás, dado que ya se había probado que las disposiciones del reglamento impugnadas por Venezuela no incluyen medidas de ejecución, el TJ afirma que Venezuela está efectivamente legitimada para impugnar aquellas disposiciones del reglamento sin tener que demostrar si le afectan individualmente.

\section{LA PRESENCIA DEL DERECHO INTERNACIONAL EN LAS ARGUMENTACIONES: LO QUE EL TJ NO ENTRÓ A CONSIDERAR}

Como ya se ha apuntado, existe una clara presencia del derecho internacional en las argumentaciones invocadas por el Consejo, algunos Estados y la Comisión, en las que se alude a la relación entre la personalidad jurídica internacional y la calificación como persona jurídica a efectos del 263, así como al principio de reciprocidad y al de inmunidad, e incluso a la costumbre internacional. Sin embargo, el TJ no se ha acogido a ninguno de esos argumentos. La razón seguramente está en que, como veremos a continuación, no parecen tener cabida en el asunto en cuestión. Sin embargo, el AG sí ha puesto de relieve su postura sobre algunos de esos principios, en ocasiones con más acierto que en otras.

Sobre la primera cuestión, el Consejo defendió que la condición de sujeto de derecho internacional público no confiere un derecho automático a

\footnotetext{
Auto del Tribunal General de 6 de septiembre de 2011, Inuit Tapiriit Kanatami y otros/ Parlamento y Consejo, T-18/10, EU: T:2011:419, apdo. 56 y la Sentencia del Tribunal General de 25 de octubre 2011, Microban/ Comisión, T-262/10, EU: T:2011:623, apdo. 21.
} 
interponer un recurso ante los órganos jurisdiccionales de los demás Estados, por lo que un tercer Estado no miembro no puede incluirse en el concepto de "persona jurídica» de la citada disposición salvo que el ordenamiento jurídico de la UE le haya conferido derechos específicos en virtud de un acuerdo celebrado por esta organización, una situación que no se plantea en el presente caso. En cambio, el AG consideró que el art. 263 TFUE, párr. 4, no diferencia entre los distintos tipos de personas jurídicas, por lo que «dado que la recurrente goza de personalidad jurídica merced a su condición de Estado soberano, debe ser considerada como "persona jurídica" en el sentido del art. 263 TFUE, párrafo cuarto» ${ }^{28}$.

En cuanto a la segunda cuestión, planteada por el Consejo y algunos Estados (Grecia, Polonia, Eslovenia y Suecia) en el sentido de que permitir el acceso de Estados terceros a los tribunales de la UE colocaría a esta organización en una situación de desventaja debido a la posible falta de reciprocidad con respecto del acceso a la UE a los tribunales de Estados terceros, hay que decir que el TJ apenas atiende a esta cuestión, limitándose a decir que «las obligaciones de la Unión de velar por el respeto del valor del Estado de Derecho no pueden supeditarse en modo alguno a un requisito de reciprocidad por lo que respecta a las relaciones de la Unión con Estados terceros» ${ }^{29}$. En la misma línea, el AG afirmó que «la justicia, la equidad y la tutela judicial efectiva son sellos distintivos de la tradición democrática, que constituye una característica básica y esencial de los 27 Estados miembros y de la Unión Europea por igual ${ }^{30}$ por lo que - y en línea también con lo alegado por Venezuela, Bélgica, Bulgaria, Alemania, Estonia, Letonia, Lituania y Países Bajos- afirmó que el respeto a un valor fundamental de la UE como es el Estado de Derecho no podía supeditarse a un requisito de reciprocidad en relaciones de esta organización con Estados terceros. En ese sentido, no se puede obviar que, como principio de derecho internacional, este también debe considerarse como uno de los principios rectores de las relaciones exteriores de la UE, lo que significa que esta organización está sometida a las normas de derecho internacional en ese ámbito concreto, incluyendo así el respeto al principio de la reciprocidad. Sin embargo, en el presente asunto las relaciones de la UE no se rigen por el derecho internacional público puesto que no se trata de la discusión de un acuerdo internacional celebrado por aquella organización, sino de si la UE debe asumir la posibilidad de que un tercer Estado plantee una demanda ante

28 Conclusiones del abogado general Hogan, Venezuela/Consejo, C-872/19 P, punto 70.

29 Sentencia del Tribunal de Justicia de 22 de junio de 2021, Venezuela/Consejo, C-872/19 P, apdo. 52.

30 Conclusiones del abogado general Hogan, Venezuela/Consejo, C-872/19 P, punto 87. 
el TJUE. Es más, el AG llega a apuntar que incluso en el caso de que fuera de aplicación y se probase esa falta de reciprocidad en lo relativo al acceso a los tribunales de un Estado tercero

no por ello la Unión quedaría exenta de sus obligaciones de garantizar los más elevados niveles democráticos, de respeto del Estado de Derecho y de resolución de los litigios por un sistema judicial independiente [...] el respeto del Estado de Derecho y del principio de tutela judicial efectiva no se basa en ningún concepto de reciprocidad ni ha de ser negociado ni acordado por vía diplomática ni sometido a obligaciones convencionales recíprocas ${ }^{31}$.

En tercer lugar, el Consejo alegó que el principio de inmunidad del Estado que rige en el derecho internacional se opone a que terceros Estados inicien este tipo de procedimientos ante los tribunales de la UE. El Consejo subrayó que las relaciones entre la UE y otros Estados no miembros se rigen por las normas de derecho internacional público, en las que los sujetos no tienen un derecho automático a interponer recursos ante los órganos jurisdiccionales de otros Estados, puesto que estas relaciones se basan en el principio de consentimiento. De esta forma, el Consejo concluía: «Esto es coherente con la doctrina de la inmunidad soberana, según la cual los sujetos de Derecho internacional no pueden regular, mediante sus normas internas, el comportamiento de otros sujetos de Derecho internacional $\aleph^{32}$. Sobre esta cuestión, el TJ tampoco se va a pronunciar — aquí ni siquiera sucintamente— quizás porque también ha tenido en consideración las reflexiones del AG en sus conclusiones sobre la doctrina de inmunidad soberana, que llevan a desmontar esa alegación de aquella institución. Para el AG, no tiene cabida invocar el principio de inmunidad del Estado para tratar de limitar la legitimación activa de Estados terceros en un recurso de anulación puesto que este principio se refiere a la prerrogativa de un Estado de no ser demandado ni enjuiciado por los órganos jurisdiccionales de otro Estado y por ello atañe en exclusiva a su legitimación pasiva. Este principio no puede extenderse, tal como se desprende del art. 8 de la Convención de las Naciones Unidas sobre las inmunidades jurisdiccionales de los Estados y sus bienes, a que los Estados no puedan acudir como legitimados activos ante tribunales extranjeros. Esta idea además, según el AG viene reforzada por la jurisprudencia de la CIJ en el asunto Inmunidades jurisdiccionales del Estado (Alemania/Italia), donde confirmó que la citada doctrina

31 Conclusiones del abogado general Hogan, Venezuela/Consejo, C-872/19 P, punto 87.

32 Sentencia del Tribunal de Justicia de 22 de junio de 2021, Venezuela/Consejo, C-872/19 P, apdo. 29. 
tiene como objeto proteger a los Estados frente a posibles demandas de otros Estados (Stewart, 2005: 194; Hafner y Köhler, 2004: 3-49).

En este orden de ideas, el argumento es válido asimismo frente a la postura de la Comisión Europea, antes apuntada, que, también atendiendo a la doctrina de inmunidad de jurisdicción, entendió que los Estados terceros podrían considerarse como "persona jurídica», cuando los actos que realizase ese Estado tercero pudieran catalogarse como iure gestionis puesto que, en virtud del derecho internacional, un Estado no puede ser sometido a la jurisdicción de otro Estado en relación con actos de poder público. Sin embargo, si como antes se dijo el principio de inmunidad soberana no se extiende a los supuestos en que terceros Estados puedan interponer recursos ante los tribunales de otro Estado, la distinción de este tipo de actos para considerar que en algunos casos podría invocarse resulta irrelevante, puesto que esa diferenciación tiene como finalidad actuar como un criterio delimitador para activar o no el principio de inmunidad soberana.

Finalmente, el AG se apoya también en que el TJ en su jurisprudencia se inspira en ocasiones en los principios y costumbres del derecho internacional público para defender que «la interpretación del artículo 263 TFUE, párrafo cuarto, se atenga al Derecho internacional público consuetudinario en cuanto a lo que debe entenderse por "persona jurídica", admitiendo al propio tiempo, por supuesto, que estos términos poseen en el Derecho de la Unión un significado autónomo que ha de determinar el Tribunal de Justicia ${ }^{33}$. Según el AG, dichas costumbres y principios requieren que los tribunales de la Unión estén abiertos a recursos interpuestos por otros Estados soberanos como personas jurídicas con capacidad para interponerlos ${ }^{34}$. Sin embargo, sobre esta cuestión se ha dicho que la posición del AG no es convincente ni prudente (Kassoti, 2021). No resulta convincente puesto que no existe ninguna norma de derecho internacional consuetudinario que permita a los Estados iniciar acciones judiciales ante los órganos jurisdiccionales de otros Estados. Tampoco resulta prudente puesto que para darle valor a la práctica de derecho internacional el AG se refiere a la práctica seguida por un Estado, Estados Unidos, emanada de la jurisprudencia de su Tribunal Supremo sin que pueda justificar su existencia en virtud del derecho internacional general. Como se ha afirmado, «while the principle of comity is distinct from international law, it undoubtedly remains an internationally oriented principle that is important in shaping a State's relations with the outside world» (ibid.).

33 Conclusiones del abogado general Hogan, Venezuela/Consejo, C-872/19 P, punto 71.

34 Ibid., punto 72. 


\section{LA DIVERGENTE ARGUMENTACIÓN POR EL TG Y EL TJ DEL REQUISITO DE LA AFECTACIÓN DIRECTA CON RESPECTO A VENEZUELA}

Como ya hemos apuntado, la argumentación del TG en el asunto T-65/18 giró en exclusiva en torno a la interpretación del requisito de la afectación directa. A continuación, comprobaremos las posturas divergentes sobre esta cuestión mantenida por los dos grados de jurisdicción.

Ambos tribunales se remiten a su jurisprudencia reiterada, donde recuerdan en primer lugar que del art. 263 TFUE, párrafo 4, se desprende que para comprobar que la decisión objeto de recurso afecta directamente a una persona física o jurídica se requiere por un lado que la medida impugnada surta efectos directamente en la situación jurídica del demandante y, por otro, que no deje ninguna facultad de apreciación a los destinatarios encargados de su aplicación por tener esta carácter meramente automático y derivarse únicamente de la normativa de la UE sin aplicación de otras formas intermedias $^{35}$. En segundo lugar, recuerdan asimismo que para determinar que un acto produce efectos jurídicos se ha de atender a su objeto, su contenido, su alcance y su fondo, además de al contexto fáctico y jurídico en el que se adoptós $^{36}$.

Sin embargo, el TG al valorar esta primera cuestión realiza una interpretación muy restrictiva del criterio de la afectación directa con respecto a la situación jurídica de Venezuela en base a tres motivos principales por los que finalmente justifica que los arts. 2, 3, 6 y 7 del Reglamento 2017/2063 no afectan directamente a la situación jurídica de aquel Estado. En cambio, el TJ, compartiendo en gran medida las conclusiones aportadas por el AG, adoptará una postura más flexible y justificada en base al carácter novedoso del asunto, donde por primera vez un Estado tercero solicita la anulación de

35 En este sentido el TG se remite al Auto del Tribunal General de 8 de octubre de 2015, Agrotikos Synetairismos Profitis Ilias/Consejo, T-731/14, no publicado, EU: T:2015:821, apdo 26, y a la Sentencia del Tribunal General de 13 de septiembre de 2018, Almaz-Antey/Consejo, T-515/15, no publicada, EU: T:2018:545, apdo. 62. Por su parte, el TJ se refiere a los asuntos: Sentencia del Tribunal de Justicia de 5 de noviembre de 2019, BCE y otros/Trasta Komercbanka y otros, C-663/17 P, C-665/17 P y C-669/17 P, EU:C:2019:923, apdo. 103, y Sentencia del Tribunal de Justicia de 3 de diciembre de 2020, Changmao Biochemical Engineering/Distillerie Bonollo y otros, C-461/18 P, EU:C:2020:979, apdo. 58.

36 Sentencia del Tribunal General de 20 de septiembre de 2019 Venezuela/Consejo, T-65/18, apdo. 30; Sentencia del Tribunal de Justicia de 22 de junio de 2021, Venezuela/Consejo, C-872/19 P, apdo. 66. 
medidas restrictivas. De ahí que el AG llegara incluso a afirmar que el TG erró en su planteamiento de base y que debería haber partido del pronunciamiento del TJ en la sentencia $P K K y K N K /$ Consejo, donde determinó que "las normas procesales que regulan la admisibilidad de un recurso de anulación deben aplicarse adaptándose en la medida necesaria a las circunstancias del caso. [...] se trata de evitar un formalismo excesivo que equivaldría a negar toda posibilidad de interponer un recurso de anulación, cuando precisamente la entidad en cuestión ha sido objeto de medidas comunitarias restrictivas ${ }^{37}$.

En cuanto al primero de ellos, el TG se refiere al ámbito de aplicación ratione loci y entiende que el art. 20 del reglamento en cuestión circunscribe las prohibiciones de los arts. 2, 3, 6 y 7 al territorio de la UE, a las personas físicas nacionales de un Estado miembro y a las personas jurídicas constituidas con arreglo al derecho de uno de esos Estados, así como a las personas jurídicas, entidades y organismos en relación con cualquier negocio efectuado, en su totalidad o en parte, en la Unión Europea, por lo que no pueden afectar directamente a la situación jurídica de la recurrente ${ }^{38}$. El TJ parece no manifestarse sobre esta cuestión, extremo sobre el que sí se pronuncia el AG, al entender que el art. 20 del reglamento se limita a indicar el ámbito de competencias del legislador de la UE en relación con unas medidas restrictivas concretas y que «el hecho que este no se aplique a la recurrente, sobre la cual es evidente que el legislador de la UE carece de competencias, no significa necesariamente que las medidas aplicables, por ejemplo, solo en el territorio de la Unión y que son vinculantes para los nacionales de un Estado miembro, no afecten directamente a la situación de la recurrente» ${ }^{39}$.

El segundo de los motivos fue el relativo al ámbito de aplicación ratione personae, en la medida que el TG afirma que los arts. 2, 3, 6 y 7 del reglamento no imponen obligaciones a la República Bolivariana de Venezuela y que —en todo caso- podrían tener efectos indirectos sobre la demandante al suponer una limitación para proveerse de los productos y servicios en cuestión ${ }^{40}$. De nuevo el TJ no coincide con la posición del TG ya que, según este, las citadas disposiciones ponían de manifiesto claramente que las medidas restrictivas fueron adoptadas contra ese Estado. En su argumentación entiende que si bien es cierto que los arts. 2 y 3 que establecen la lista de prohibiciones y se refieren

37 Conclusiones del abogado general Hogan, Venezuela/Consejo, C-872/19 P, punto 105, donde se refiere al apartado 114 de la Sentencia del Tribunal de Justicia de 18 de enero de 2007, PKK y KNK/Consejo, C-229/05 P, EU:C:2007:32. Sentencia del Tribunal General de 20 de septiembre de 2019 Venezuela/Consejo, T-65/18, apdo. 32 .

39 Conclusiones del abogado general Hogan, Venezuela/Consejo, C-872/19 P, punto 113.

40 Sentencia del Tribunal General de 20 de septiembre de 2019 Venezuela/Consejo, T-65/18, apdo. 33 . 
a «cualquier persona física o jurídica, entidad u organismo sitos en Venezuela o para su utilización en este país», también incluyen al Gobierno, organismos públicos, compañías y agencias de Venezuela y a cualquier persona o entidad que actúe en su nombre o por indicación suya, como se deduce explícitamente de los art. 6 y 7 del citado reglamento ${ }^{41}$. Por ello, llega a la conclusión de que las decisiones impugnadas se dirigen específicamente a Venezuela y a las diversas emanaciones de dicho Estado puesto que aquellas intentan garantizar que Venezuela no obtenga determinados productos y servicios de ninguna de las personas mencionadas en el art. 20 del Reglamento.

En tercer lugar, el TG se refirió al asunto Almaz-Antey/Consejo, donde el TG había argumentado que las disposiciones impugnadas contempladas en una decisión establecen prohibiciones que se aplican en primer lugar a las entidades de crédito y otros organismos financieros establecidos en la UE, puesto que los actos adoptados por las instituciones de la UE no están, por regla general, destinados a aplicarse fuera del territorio de la Unión Europea. Sin embargo, ello no significaba que las entidades afectadas por las disposiciones de una decisión no estén directamente afectadas por las medidas restrictivas que se les aplican; esto equivaldría a considerar que, «even in cases of individual fund freezes, the listed persons subject to the restrictive measures are not directly concerned by such measures, given that it is primarily for the EU Member States and the natural or legal persons under their jurisdiction to apply them ${ }^{42}$. No obstante, el TG consideró que la citada sentencia no era aplicable al asunto en cuestión sobre la base de dos argumentos. El primero, que en el asunto Almaz-Antey/Consejo el acto impugnado se refería a una empresa cuyo nombre figuraba expresamente en el anexo de la decisión y en este caso los arts. 2, 3, 6 y 7 del reglamento «no se referían expresa y específicamente a Venezuela, como Estado, de modo comparable» ${ }^{43}$. El segundo, que Venezuela no podía equipararse a un operador como la demandante en el asunto T-515/15, puesto que Venezuela «dispone, como Estado, de un ámbito de actuación que se caracteriza por una extrema diversidad y que no puede reducirse a una actividad específica " ${ }^{44}$. Además, como se desprendía

41 Sentencia del Tribunal de Justicia de 22 de junio de 2021, Venezuela/Consejo, C-872/19 P, apdo. 66. Véase el punto 110 de las Conclusiones del abogado general Hogan, Venezuela/Consejo, C-872/19 P.

42 Sentencia del Tribunal General de 13 de septiembre de 2018, Almaz-Antey/Consejo, T-515/15, no publicada, EU: T:2018:545, apdo. 65.

43 Sentencia del Tribunal General de 20 de septiembre de 2019 Venezuela/Consejo, T-65/18, apdo. 36 .

44 Sentencia del Tribunal General de 20 de septiembre de 2019 Venezuela/Consejo, T-65/18, apdo. 37. 
—entre otros- del asunto Inuit Tapiriit Kanatami y otros/Parlamento y Consejo, las prohibiciones que establece el reglamento en sus arts. 2 y 3 no pueden afectar directamente a la situación de operadores que no están presentes en los mercados de que se trate ${ }^{45}$.

Pues bien, la cuestión de si Venezuela debía ser identificada en el anexo del acto en cuestión para que se aplicara por analogía el asunto Almaz-Antey/Consejo parece irrelevante, ya que de admitir lo anterior supondría que el derecho a emprender acciones judiciales por la vía del 263 TFUE quedaría supeditada a que se identificara a esa persona o entidad en el anexo, no siendo suficiente que fuera mencionada en el cuerpo de disposición. Así, lo importante es la incidencia de las disposiciones impugnadas en la situación jurídica del recurrente, no la forma concreta en la que adopten dichas prohibiciones. Después, en lo relativo a que Venezuela no podría equipararse a un operador económico, el TJ consideró que, a efectos de determinar el criterio de la afectación directa, tampoco importaba que la actividad del recurrente no se limitase a los de un operador económico presente en los mercados puesto que, como añade el AG, del tenor del art. 263 TFUE, párrafo cuarto ${ }^{46}$, no podía desprenderse que se imponen condiciones relativas al estatuto o capacidad de la persona ${ }^{47}$. Lo que finalmente lleva al TJ a afirmar que «no es necesario distinguir si esas operaciones comerciales se efectúan iure gestionis o iure imperii, ya que tal distinción no puede inferirse ni del art. 263, párr. cuarto, ni de ninguna otra disposición de la Unión» ${ }^{48}$.

En síntesis, se puede afirmar que con respecto a los efectos de las disposiciones impugnadas en la situación jurídica del recurrente el TG se decanta por una interpretación «sumamente artificial e indebidamente formalista ${ }^{49}$, mientras que el TJ ha preferido una interpretación mucho más flexible.

\section{LAS POSIBLES CONSECUENCIAS DE LA SENTENCIA}

En contra de lo alegado por el Consejo y algunos Estados miembros, cabe estimar que incluir a Estados terceros contra los que van dirigidas medidas restrictivas de la UE en el concepto de "persona jurídica» según el

45 Sentencia del Tribunal General de 20 de septiembre de 2019 Venezuela/Consejo, T-65/18, apdo. 34 a 41.

46 Sentencia del Tribunal de Justicia de 22 de junio de 2021, Venezuela/Consejo, C-872/19 P, apdo. 72 .

47 Conclusiones del abogado general Hogan, Venezuela/Consejo, C-872/19 P, punto 121.

48 Sentencia del Tribunal de Justicia de 22 de junio de 2021, Venezuela/Consejo, C-872/19 P, apdo. 77.

49 Conclusiones del abogado general Hogan, Venezuela/Consejo, C-872/19 P, punto 109. 
art. 263 TFUE, párr. cuarto, aporta una garantía más con respecto de la tutela judicial efectiva, que debe ser observada por la UE y contribuye a consolidar su imagen en el exterior como una organización que consagra el Estado de Derecho como uno de sus valores fundamentales. Esto viene reforzado por la idea de que «las normas sobre la legitimación activa establecidas en el artículo 263 TFUE y, en particular, en su párrafo cuarto se basan en criterios objetivos que se establecieron en los Tratados y que han sido interpretados por los tribunales de la Unión y no en la existencia o ausencia de acuerdos recíprocos sobre la legitimación activa entre la Unión y terceros Estados ${ }^{50}$ y, por tanto, ningún otro criterio puede hacer que la UE postergue su obligación de respetar los valores sobre los que se asienta.

Habrá que esperar para comprobar si en un futuro esto también podría suponer un cambio en el enfoque de la UE con respecto de la elección de medidas restrictivas autónomas, puesto que si se ha reconocido la legitimación activa a Estados terceros para impugnar medidas de ejecución de decisiones como las relativas a la interrupción o la reducción de las relaciones económicas y financieras contra un tercer Estado, a partir de ahora la UE podría inclinarse por la aplicación de regímenes de sanciones temáticos (Finelli, 2021). En este sentido, y dado que las medidas restrictivas individualizadas no han sido el objeto de controversia en el presente asunto, quizás la reciente aprobación de un nuevo régimen global de sanciones en materia de derechos humanos ${ }^{51}$ permitiría superar un régimen de medidas dirigidas a Estados terceros «ante el riesgo de que se produzcan [...] nuevas violaciones o abusos de los derechos humanos ${ }^{52}$ y priorizar aquellas relativas al bloqueo de fondos y recursos económicos de personas físicas o jurídicas, entidades u organismos que sean responsables de violaciones o abusos graves de los derechos humanos. No obstante, también habrá que esperar para comprobar en qué medida las sanciones individuales pueden producir cambios en las conductas de ciertos sujetos para prevenir futuras violaciones graves de los derechos humanos.

En todo caso, parece necesario recordar que una cosa es que se esté reconociendo a un tercer Estado la posibilidad de ser considerado como persona jurídica a efectos del art. 263 TFUE, párr. cuarto, y otra bien distinta

50 Conclusiones del abogado general Hogan, Venezuela/Consejo, C-872/19 P, punto 122.

51 Reglamento (UE) 2020/1998 del Consejo, de 7 de diciembre de 2020, relativo a medidas restrictivas contra violaciones y abusos graves de los derechos humanos, (DO L I 410, de 7 de diciembre de 2020, p. 1).

52 Decisión (PESC) 2017/20174 del Consejo de 13 de noviembre de 2017, relativa a medidas restrictivas habida cuenta de la situación en Venezuela, (DO L 295, de 14 de noviembre de 2017 , p. 60). 
es que se esté eximiendo a estos sujetos de cumplir con los demás requisitos de admisibilidad contemplados en la disposición citada o, incluso, que se esté posibilitando a Estados terceros discutir la pertinencia, que no la legalidad, de las medidas restrictivas adoptadas por la UE.

\section{Bibliografía}

Condinanzi, M. y Mastroianni, R. (2009). Il ricorso per annullamento. En M. Condinanzi y R. Mastroianni. Il contenzioso dell'Unione Europea (pp. 109-121). Torino: Giappichelli Editore.

Cortés Martín, J. M. (2014). Sobre el Locus Standi del particular en el control de la legalidad. Revista General de Derecho Europeo, 34, 1-36.

De Faramiñán Gilbert, J. M. (2007). El Contencioso de la Unión Europea. El control de la legalidad en la Unión Europea. En J. M. Beneyto Pérez. Tratado de derecho y politicas de la Unión Europea (pp. 321-426). Madrid: Aranzadi Thomson Reuters.

Finelli, F. (2021). Does A Third State Have Legal Standing To Challenge EU Restrictive Measures? The Court Of Justice Says 'Yes'. EU Law Analysis [blog], 1-8-2021. Disponible en: https://bit.ly/3pesw46.

Hafner, G. y Köhler, U. (2004). The United Nations Convention on Jurisdictional Immunities of States and Their Property. Netherlands Yearbook of International Law, 35, 3-49. Disponible en: https://doi.org/10.1017/S0167676804000030.

Jiménez García, F. (2017). Medidas restrictivas en la Unión Europea: entre las sanciones y el unilateralismo europeo. En C. Martínez Capdevila y E. J. Martínez Pérez (dirs.). Retos para la acción exterior de la Unión Europea (pp. 509-534). Valencia: Tirant lo blanch.

Kassoti, E. (2021). State Immunity, Comity and the Question of Legal Standing of a Third Country before the CJEU: The Opinion of AG Hogan in Case C-872/19 P Venezuela v Council. EU Law Analysis [blog], 9-4-2021. Disponible en: https://bit.ly/30JD7Kt.

Martín Baumeister, B. (2004). El reconocimiento de la legitimación activa a las regions europeas en recursos de anulación bajo el Art. 230.4 CE: elemento de afectación individual. Gaceta Jurídica Europea, 234, 29-45.

Stewart, D. P. (2005). The UN Convention on Jurisdictional Immunities of States and their Property. American Journal of International Law, 99(1), 194-211. Disponible en: https://doi.org/10.2307/3246098. 